\title{
The cultured primary hepatocyte and its application in toxicology
}

\author{
Peter M. Eckl and Nikolaus Bresgen
}

University of Salzburg, Institute of Genetics and General Biology, Hellbrunnerstr. 34, A-5020 Salzburg, Austria

\begin{abstract}
Summary
The liver is the main organ involved in the metabolism of xenobiotic (foreign) compounds. The responsible enzymatic systems are the cytochromes P450 (mixed function oxidases or phase I reactions) and enzymes coupling larger water soluble groups to the substrate (phase II reactions). Especially during phase I reactions, highly reactive metabolites can be formed capable of interacting with DNA and causing mutations. On the other hand reactive xenobiotics may be detoxified. Therefore, the primary parenchymal liver cell (hepatocyte) appears to be the optimal and most reliable in vitro system for the determination of mutagenicity/genotoxicity. Since however, primary hepatocytes are proliferatively quiescent, a culture system had to be developed allowing for proliferation enabling the determination of induced changes at the chromosomal level. This paper summarizes the special features of primary hepatocytes, the findings on in vitro proliferation and the application of hepatocyte cultures for in vitro and ex vivo/in vitro toxicological testing.
\end{abstract}

Keywords: hepatocytes - primary culture - cytogenetics - cytotoxicity - genotoxicity

\section{INTRODUCTION}

Numerically, the liver consists of $60-65 \%$ of polyhedrally shaped parenchymal cells, the so called hepatocytes and the sinusoidal cells which account for about $40 \%$ and comprise the phagocytotically active Kupffer cells, endothelial cells, lipocytes, smooth muscle cells and others. Since hepatocytes are larger than the other cells they constitute about 80 to $95 \%$ of the total liver volume (Berry et al. 1991).

Hepatocytes contain at least one prominent nucleus, numerous mitochondria (approximately $25 \%$ of the cell volume), lysosomes, peroxisomes etc., but the largest fraction of the volume is occupied by the smooth and rough endoplasmic reticulum where the metabolism of xenobiotic substances mainly takes place. Xenobiotic metabolism is classified into two groups. Phase I reactions include oxidations, reductions and hydrolyses, and are executed by the cytochrome P450 enzyme system consisting of multiple forms of cytochrome P450 with overlapping substrate specificity and a NADPH dependent cytochrome $\mathrm{P} 450$ reductase. Phase II reactions are conjugation reactions with large water soluble groups, i.e. glutathione, glucuronate, sulphate. Therefore, if a lipophilic xenobiotic substance enters the liver the phase I enzymes will introduce a functional group, usually a hydroxyl group which then is conjugated to large hydrophilic functions by the phase II enzymes to obtain water soluble metabolites (Guillouzo 1986, Kremers 1986).

In the course of this so-called biotransformation, lipophilic xenobiotic substances may not only become more water soluble and thus more readily excretable, but also highly reactive metabolites can be formed from a non-reactive substance. Typical examples include the environmental mutagens/carcinogens aflatoxin $\left(\mathrm{AFB}_{1}\right)$ - a mycotoxin produced by the mould Aspergillus flavus - and benzo(a)pyrene, a polycyclic aromatic hydrocarbon released during diverse combustion processes. These are biotransformed to epoxides capable of interacting with DNA and inducing mutations. Mutagens/carcinogens which require metabolism to produce the ultimate mutagens are referred to as indirectly acting mutagens or promutagens/procarcinogens, and a large portion of the mutagens/carcinogens present in 
the environment, either naturally or due to human activities, belongs to this group.

In order to identify these it is necessary to utilise the xenobiotic metabolising capacity of hepatocytes which was originally carried out by applying liver microsomal fractions to a given in vitro test system, i.e. bacteria (Maron and Ames 1983) or mammalian cell lines (Natarajan et al. 1976). However, under these conditions metabolites can be formed different from those found in vivo (DeFlora 1978), and/or differences in the amount of reactive metabolites entering the indicator cell due to permeability can be expected. It has been demonstrated that primary hepatocyte cultures retain the capacity to metabolize chemical carcinogens (Strom and Michalopoulos 1982, Strom et al. 1983) and can be considered to be an ideal and highly sensitive test system for the evaluation of the genotoxic potential of mutagens/carcinogens. Therefore, reproducible culture conditions had to be developed allowing the proliferation of primary hepatocytes and thus the determination of damages to the hepatocyte genome.

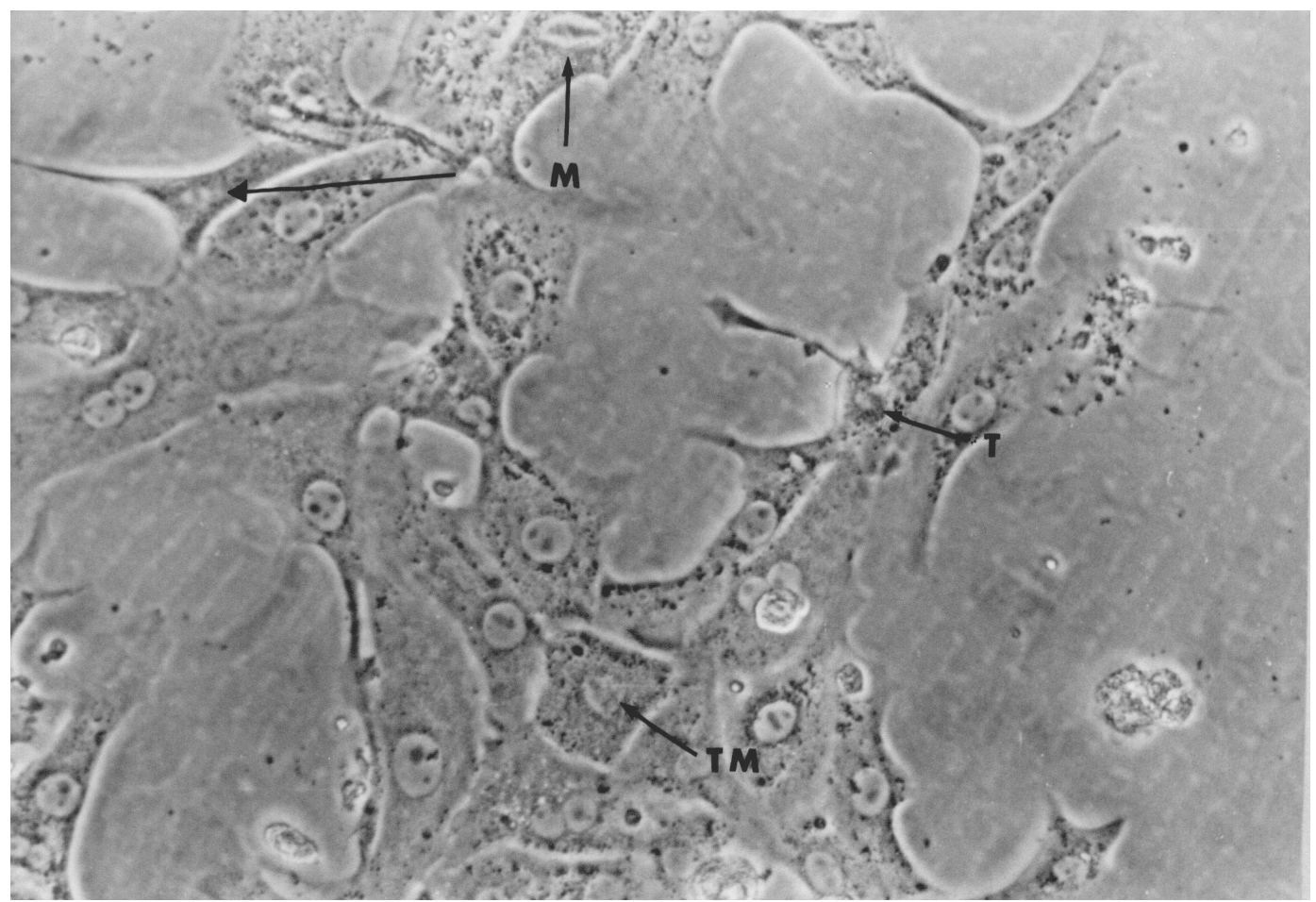

Fig. 1. Section of a proliferating culture of primary hepatocytes. M: mitosis, TM: tripolar mitosis

\section{Proliferation of primary hepatocytes}

Primary hepatocytes are highly differentiated cells and proliferatively quiescent $\left(\mathrm{G}_{0}\right)$. Proliferation can be stimulated under certain culture conditions such as extracellular matric components (Reid et al. 1986), low cell densities (Michalopoulos et al. 1982), mitogens and co-mitogens in appropriate concentrations (Michalopoulos 1990) and subphysiological calcium concentrations (Eckl et al. 1987a). The growth of hepatocytes in a controlled environment as defined by the conditions of a serum free primary culture offers the additional advantage that growth inhibitory effects of growth factors such as $\mathrm{TGF} \beta_{1}$ present in the serum are avoided.
Based upon these observations the following culture conditions were elaborated:

a) Collagen coating of the culture dishes: hepatocytes attach better and maintain differentiated functions for longer periods when grown on collagenous substrates (Hirata et al. 1983);

b) MEM medium with non-essential amino acids further supplemented with pyruvate, serin and aspartate;

c) Serum free medium: to avoid the above mentioned action of growth inhibitory factors and to have an optimally controlled culture environment;

d) Sub-physiological calcium concentration of $0.4 \mathrm{mM}$ : At this concentration the 
proliferation rate peaks as evidenced by the analysis of second division metaphases (Eckl et al 1987a);

e) Epidermal growth factor at a concentration of $40 \mathrm{ng} / \mathrm{ml}$;

f) Insulin at a concentration of $10^{-7} \mathrm{M}$, and

g) Plating at cell densities of 5 to 20000 viable cells per $\mathrm{cm}^{2}$ : At lower and higher concentrations the proliferation rate drops (Eckl et al. 1987a).

Under these culture conditions the labelling index (LI, percentage of cells having synthesized DNA) increases continually up to $72 \mathrm{~h}$ after proliferative stimulation with EGF. At this time approximately $70 \%$ of the cells are labelled (Eckl and Raffelsberger 1997). With longer times no further significant increase is observed. When analysing the mitotic index (MI, the percentage of mitotic figures at a given time point) the first mitoses are observed 24 hours after proliferative stimulation. The MI rapidly increases with culture times $>24$ hours and peaks after 48 hours. At this time point about $2 \%$ mitotic figures are observed (Fig. 1). Differential staining further revealed that the cells undergo up to three subsequent mitoses within 48 hours (Eckl et al. 1987a). At longer culture times a gradual decrease of the MI is observed.

This proliferation kinetics of primary hepatocytes enables the determination of a wide range of mutational events at the chromosomal level, ranging from endpoints requiring one completed mitosis such as the induction of chromosomal aberrations, and micronuclei to sister chromatid exchanges (SCE) requiring two subsequent rounds of replication. Nevertheless, specific features of primary hepatocytes with respect to ploidy and cell division have to be taken into consideration.

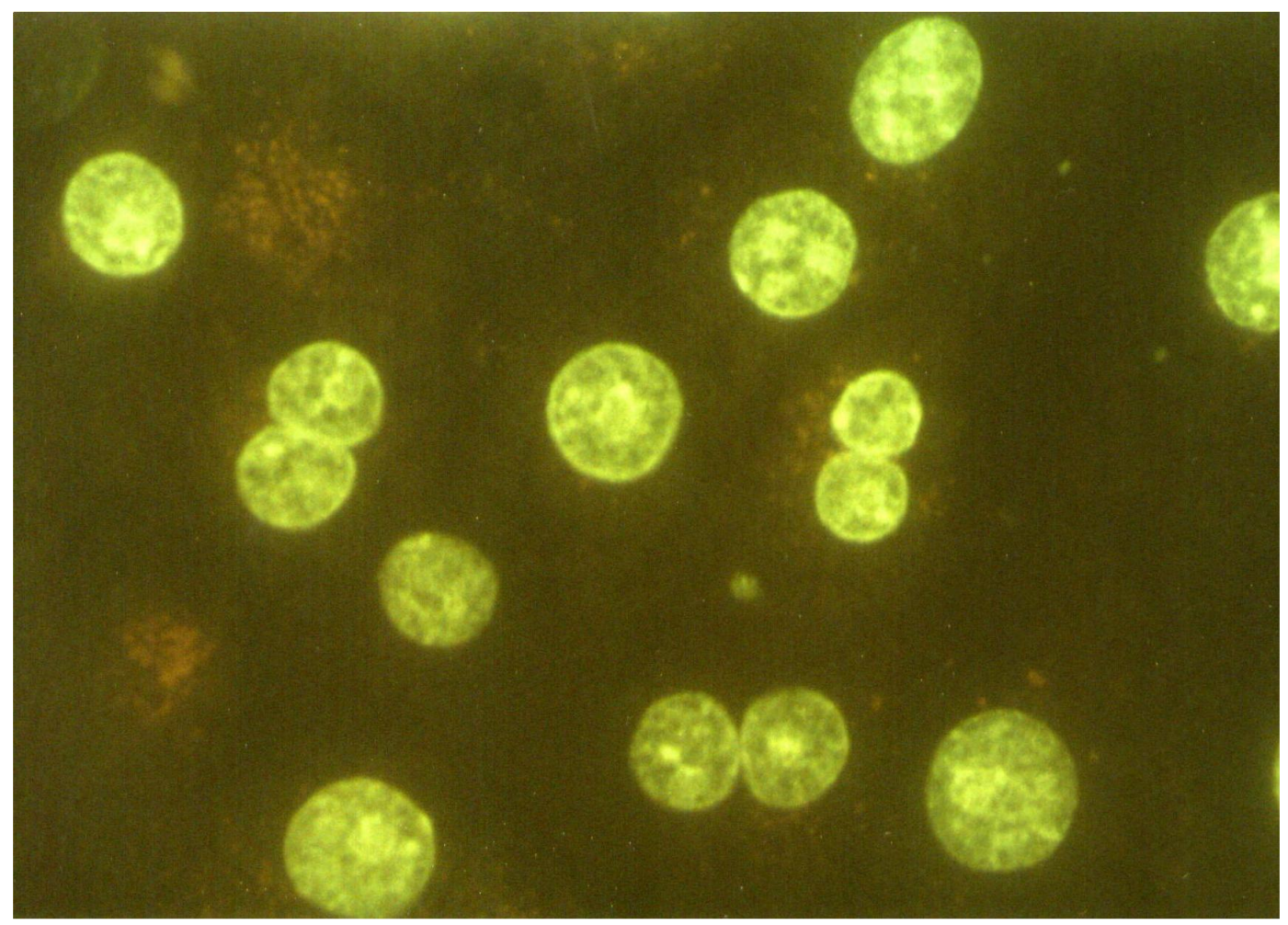

Fig. 2. Fluorescence micrograph of a DAPI stained hepatocyte culture. Note the different ploidies (sizes) of the nuclei. Binucleated cells are characterized by two nuclei of equal size close to each other.

\section{Ploidy and cell division}

Primary rat hepatocyte cultures mainly consist of tetraploid cells. Besides, there is a small fraction of diploid and octoploid cells. Approximately $30 \%$ of the cells are binucleated - cells with either two diploid or two tetraploid nuclei (Fig. 2). After proliferative stimulation with EGF the number of binucleated cells increases to a maximum value of about $50 \%$ after 79 to 120 hours. After longer cultivation times the percentage of binucleated cells decreases again. This finding can either be explained 
by fusion of mononucleated cells or that hepatocytes do not undergo cytokinesis after mitosis (Eckl 1993).

Proliferating hepatocyte cultures are characterized by the appearance of multipolar mitoses (Sattler et al. 1989, Eckl 1993). The percentage of multipolar mitotic figures was found to be about $12 \%, 80 \%$ of which are tripolar (Fig. 3) and $20 \%$ are tetrapolar. Since multipolar mitoses are only rarely found in vivo in the regenerating rat liver their appearance in vitro has to be attributed to the change from a three-dimensional to a twodimensional system where the mitotic spindles are forced into an orientation parallel to the growth surface allowing a maximum interaction of the spindles of binucleated cells and thus enhancing the probability of multipolar stages to occur.

As a consequence of multipolar mitosis a high level of chromosome loss is observed yielding approximately $70 \%$ at telophase and contributing to elevated background levels of micronuclei (Eckl 1993).

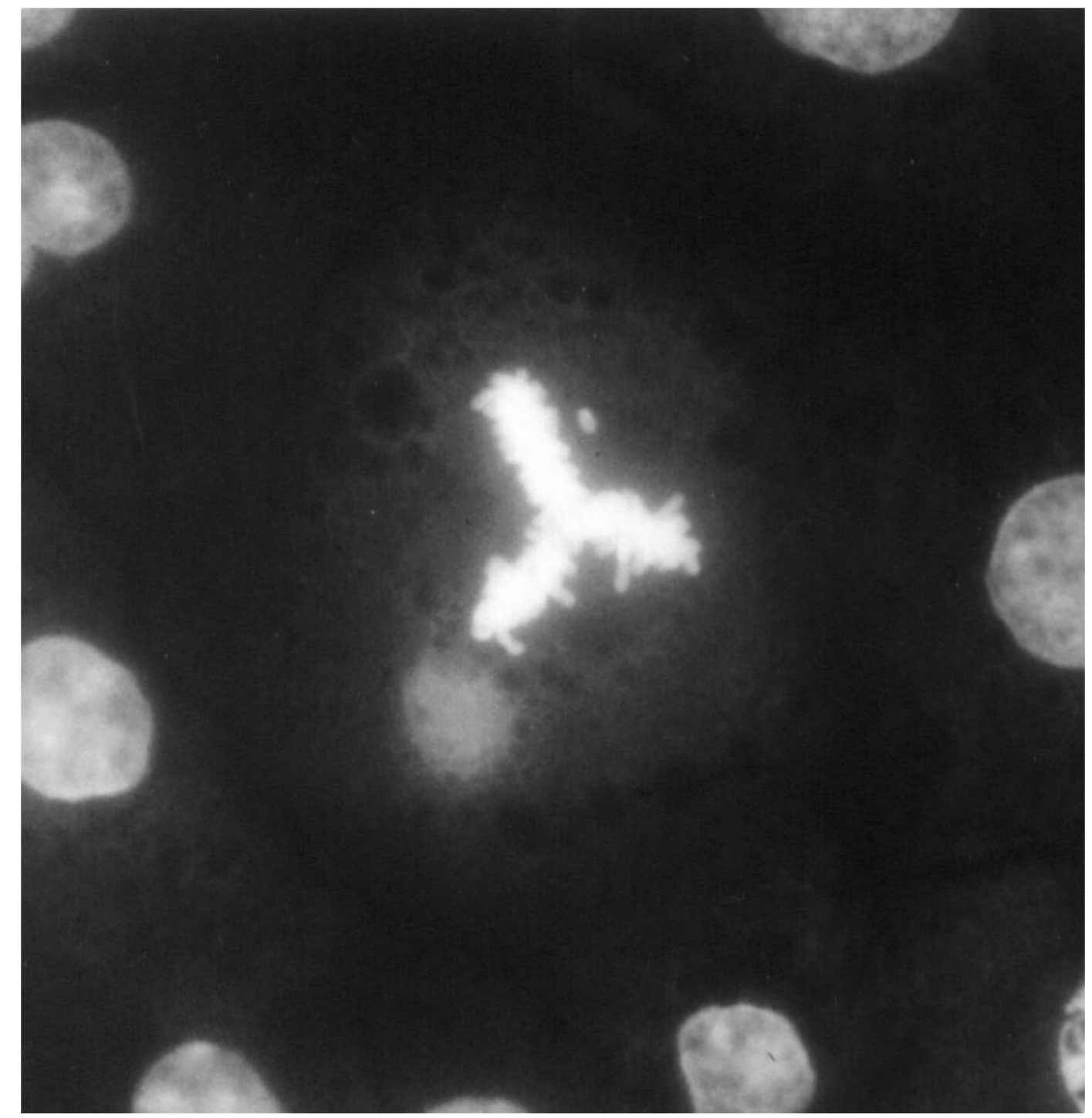

Fig. 3: Tripolar metaphase, DAPI stained

\section{Consequences for the use as a test system for genotoxicity}

Because of the features described, techniques for the determination of cytogenetic endpoints of genotoxicity cannot directly be applied to primary hepatocyte cultures. This accounts in particular for the micronucleus test (Fig. 4) which in its most developed version is based on treatment with cytochalasin B after mutagen treatment. Cytochalasin B inhibits cytokinesis and leads to the formation of binucleated cells. Since micronuclei are 
determined in binucleated cells only the portion of cells which went through division after treatment is analysed (Fenech 1993).

As pointed out above approximately $30 \%$ of primary hepatocytes are binucleated. Since there is no method to discriminate between binucleated cells originally present and those induced by cytochalasin $\mathrm{B}$, this method is not applicable. In order to obtain a similar resolution we therefore developed a method based on BrdU incorporation and subsequent immunocytochemical staining for BrdU. Micronuclei in this case are determined in labelled cells. Since hepatocytes are stimulated to proliferate after mutagen treatment, labelled micronuclei in combination with labelled nuclei can be considered to result from the treatment (Eckl and Raffelsberger 1997). Therefore non-labelled micronuclei in combination with non-labelled nuclei can be considered to have been present in the cell population originally isolated.
When comparing the results of this modified micronucleus assay with the standard assay without BrdU incorporation, similar results with respect to significance levels are obtained, indicating that this simpler version is sufficient as a test method.

With respect to the induction of sister chromatid exchanges (Fig. 5) and chromosomal aberrations (Fig. 6) fewer modifications have to be made. Here, the primary problem relates to the ploidy of the cells which means that in the case of rat hepatocytes 42 , 84 or 168 chromosomes per metaphase spread have to be counted. The results obtained therefore cannot be presented as 'events per cell'. Instead they have to be presented as 'events per chromosome', i.e. SCE per chromosome. In the case of chromosomal aberrations - in order to avoid very low numbers it is recommended that the calculation is of 'aberrations per diploid cell'.

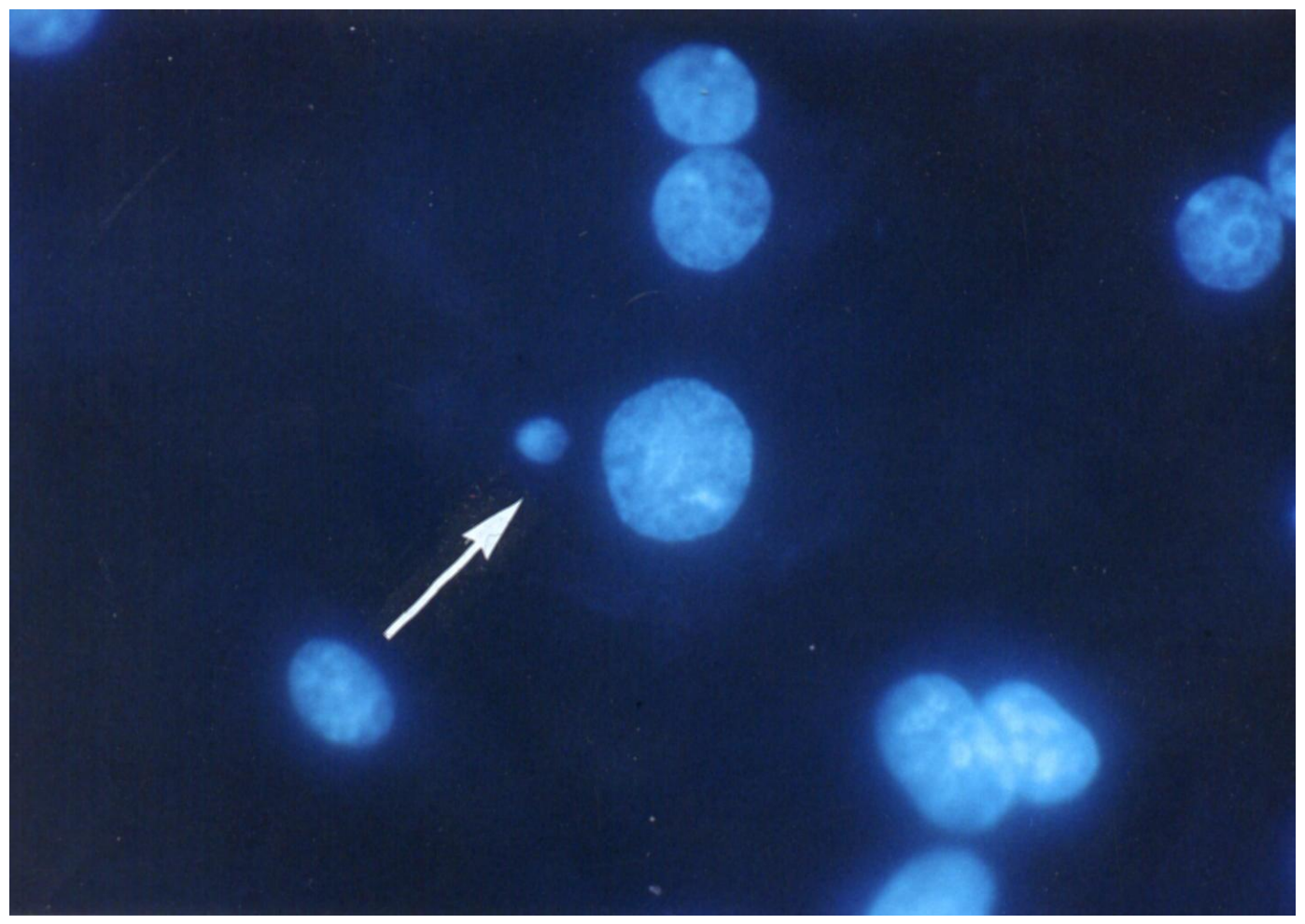

Fig. 4. Micronucleus (arrow), DAPI stained. Micronuclei resemble the nucleus in that they are also surrounded by a nuclear membrane. They usually are very small and are situated near the nucleus. This example is a medium sized micronucleus.

\section{MATERIAL AND METHODS}

\section{Materials}

Minimum essential Medium (MEM) with Earle`s salts and non-essential amino acids was obtained from Grand Island Biological Co. through Life Technologies Overseas, Vienna, Austria. Plastic culture dishes were from Greiner, Kremsmünster, Austria. EGF, collagenase and other cell culture chemicals - unless otherwise specified - were 
purchased from Sigma Chemical Co., Munich, Germany.

\section{Animals}

Female Fischer 344 rats weighing approximately $100 \mathrm{~g}$ were obtained from Harlan, Zeist, The Netherlands. They were housed in hanging cages in a temperature $\left(22 \pm 2^{\circ} \mathrm{C}\right)$ and humidity $(65 \pm 10 \%$ relative humidity) controlled room with a 12 hour light-dark cycle. Food and water was provided ad libitum. Sawdust served as bedding material. The animals were allowed to acclimatize for at least two weeks prior to hepatocyte isolation.

For the ex vivo/in vitro studies animals received the test substances either with the drinking water or food, or via tube feeding.

\section{Methods \\ Hepatocyte isolation and primary culture}

Hepatocytes were isolated from female Fischer 344 rats by the in situ two-step collagenase perfusion technique described by Michalopoulos et al. (1982). The isolated hepatocytes were plated at a density of 20000 viable cells $/ \mathrm{cm}^{2}$ on collagen-coated $60 \mathrm{~mm}$ diameter plastic culture dishes. The hepatocytes were plated according to Eckl et al. (1987a) in $5 \mathrm{ml}$ of serum free MEM supplemented with nonessential amino acids, pyruvate $(1 \mathrm{mM})$, aspartate $(0.2 \mathrm{mM})$, serine $(0.2 \mathrm{mM})$, gentamicin $(50 \mu \mathrm{g} / \mathrm{ml})$ and $0.4 \mathrm{mM}$ calcium at $37^{\circ} \mathrm{C}, 5 \% \mathrm{CO}_{2}$ and $95 \%$ relative humidity. After an incubation period of $3 \mathrm{~h}$, the medium was exchanged for freshly prepared medium supplemented as described above with the further addition of insulin $\left(10^{-7} \mathrm{M}\right)$. After replacing the medium, the cultures were returned to the incubator.

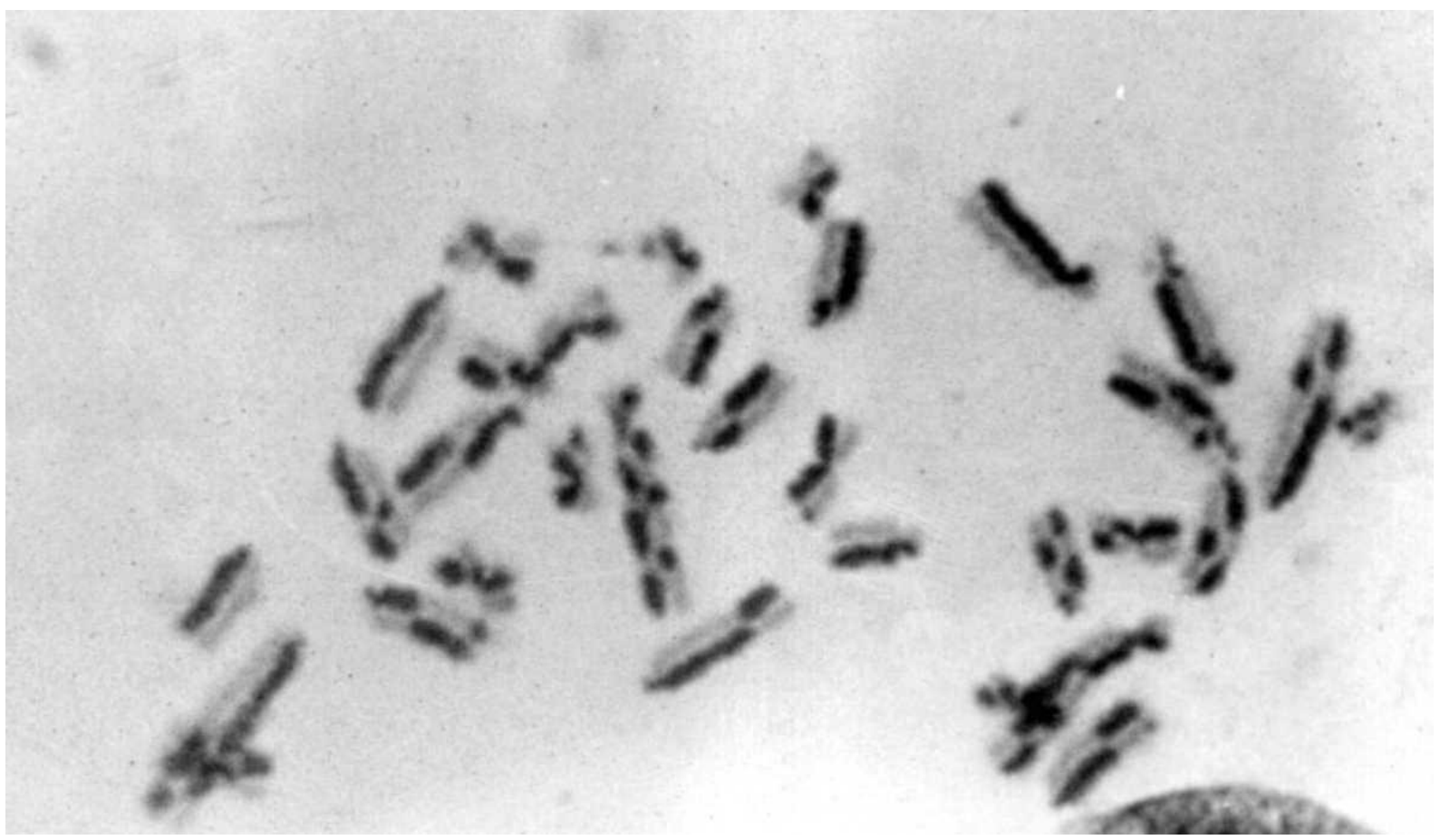

Fig. 5. Differentially stained second division metaphase showing a large number of SCEs as evidenced by the change of dark and light staining along the chromatids

\section{Treatment}

Approximately 20 hours after the initial exchange of the medium, the test substances or complex environmental mixtures (dissolved in an appropriate solvent such as water or dimethylsulfoxide = DMSO) were added to the cultures at different concentrations (5 cultures each) and incubated for
3 hours. The medium was then aspirated, the plates washed twice with fresh medium, fresh medium supplemented with EGF (40 ng/ml) and BrdU (10 $\mu \mathrm{M}$; to the cultures used for SCE analysis) added and the cultures returned to the incubator. 


\section{Cytogenetic studies}

Cytogenetic studies were performed in principle as described by Reisenbichler and Eckl (1993). After treatment the cultures were returned to the incubator. Colcemid $(0.4 \mu \mathrm{g} / \mathrm{ml})$ was added 48 hours later and the cultures incubated for a further 3 hours. The medium was replaced with $2 \mathrm{ml}$ of collagenase solution $(0.5 \mathrm{mg} / \mathrm{ml})$ and the plates returned to the incubator for an additional $10 \mathrm{~min}$. The detached cells were collected by centrifugation, treated with hypotonic solution $(0.01 \mathrm{M} \mathrm{KCl})$ for 10 min and fixed in cold methanol-glacial acetic acid overnight. Preparations were made by dropping the cell suspension in fixative on pre-cleaned glass slides followed by flame drying.

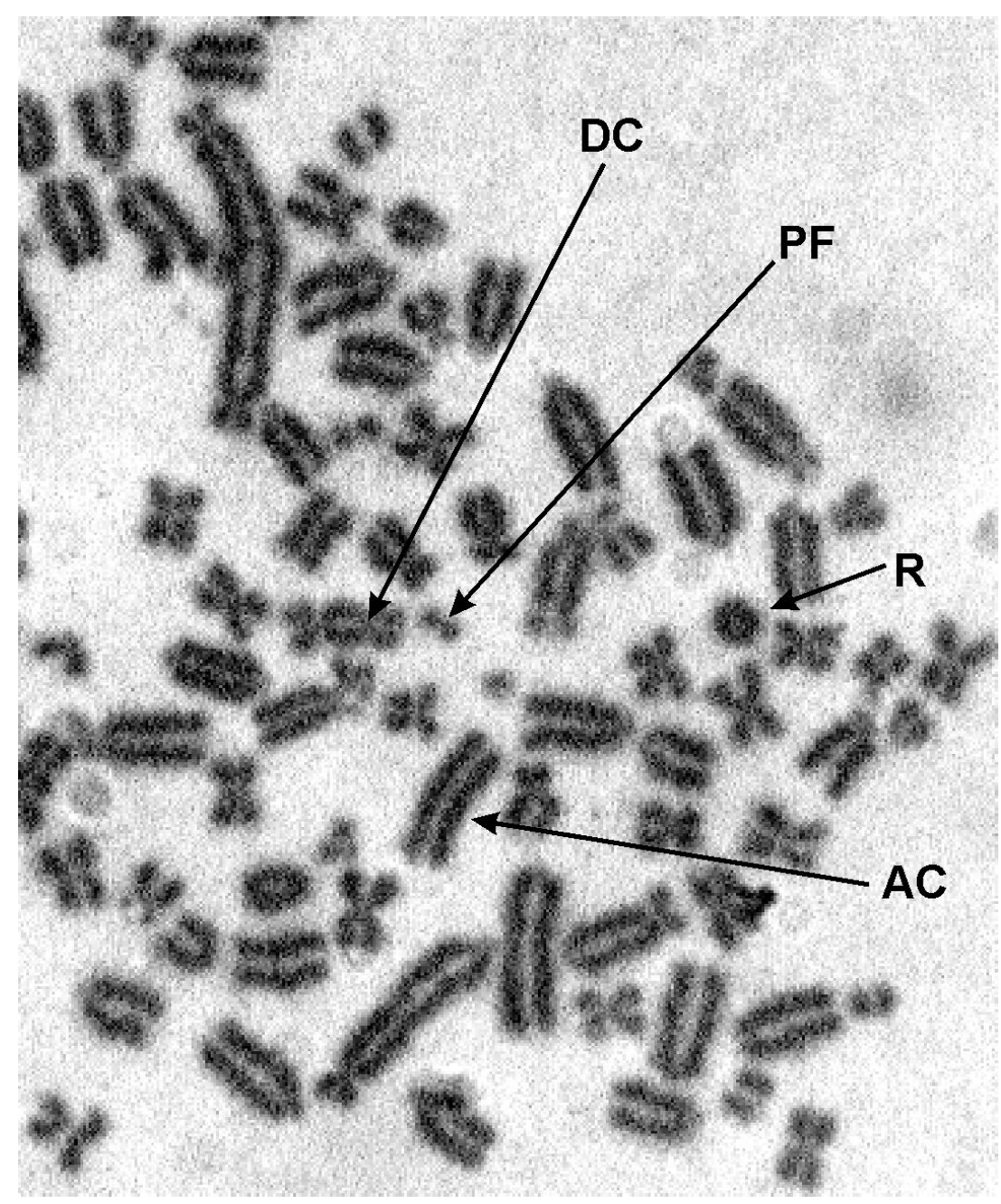

Fig. 6. Hepatocyte metaphase after treatment with MNNG showing a large number of chromosomal aberrations AC: acentric chromosome, DC: dicentric chromosome, R: ring chromosome, PF: paired fragment

The slides were stained with Hoechst 33258 $(4.5 \mu \mathrm{g} / \mathrm{ml})$ for $15 \mathrm{~min}$, rinsed with distilled water, mounted with a cover glass in PBS $(\mathrm{pH}=7)$ and exposed for $15 \mathrm{~min}$ to two 40-W blacklight lamps (General Electric, F 40 BLB Blacklight) on a warming plate kept at $30^{\circ} \mathrm{C}$. After removal of the cover glasses the slides were briefly washed with distilled water and stained in 5\% Giemsa solution. For the determination of SCE, 20 well spread second division metaphases were scored; for the determination of chromosomal aberrations 20 well spread first division metaphases.

For the determination of the mitotic index, the percentage of necrotic and apoptotic cells and the percentage of cells with micronuclei, the cultures were fixed 51 hours later in cold methanol-glacial acetic acid (3:1) for $5 \mathrm{~min}$ on the Petri dishes, rinsed with distilled water for 2 min and air dried. The fixed cells were stained with DAPI $(0.2 \mu \mathrm{g} / \mathrm{ml}$ McIlvaine citric acid $-\mathrm{Na}_{2} \mathrm{HPO}_{4}$ buffer, $\mathrm{pH}=7$ ) for 
$30 \mathrm{~min}$, briefly rinsed with distilled water and mounted in glycerol. To determine the number of cells with micronuclei and the endpoints of cytotoxicity, 1000 cells per Petri dish were analysed under the fluorescence microscope.

\section{APPLICATIONS}

In vitro testing

Primary cultures of adult rat hepatocytes were first tested for their suitability to assess SCE inducing DNA damage by mutagens and promutagens (Eckl et al. 1987b). Compared to other genotoxicity assays which utilize the metabolizing activity of liver cells or microsomes this system proved to be at least one order of magnitude more sensitive. For example Aflatoxin $\mathrm{B}_{1}$ induced significantly elevated levels of SCE even at a concentration of $10^{-12} \mathrm{M}$.

Hepatocytes appear to be especially sensitive to lipid peroxidation products induced by oxidative stress, in particular to 4-hydroxyalkenals (Eckl and Esterbauer 1989, Eckl et al. 1993a). This observation can also explain the elevated background levels of different endpoints of genotoxicity (Eckl et al. 1987b) which can be reduced to about $50 \%$ by supplementation of the medium with the antioxidant vitamins $\mathrm{C}$ and $\mathrm{E}$ (Eckl and Raffelsberger 1997), and is due to the higher oxygen partial pressure in primary culture compared to in vivo.

Additionally, hepatocytes proved to be a valuable test system when other assays gave conflicting results. For example, peroxisome proliferators (plasticizers such as phthalates and hypolipidemic drugs such as ciprofibrate) are potent liver carcinogens, however, different test systems failed to prove a genotoxic potential. Primary cultures of hepatocytes on the other hand demonstrated a genotoxic potential for this class of carcinogens (Reisenbichler and Eckl 1993) potentially indicating a higher reliability of the results, at least for a liver associated risk.

From the results obtained thus far with pure compounds, primary hepatocytes can be considered to be the most sensitive assay for the determination of genotoxicity.

Because of this high sensitivity, primary hepatocytes were further tested for their use in determining the genotoxic potential of complex environmental mixtures, primarily surface and ground water samples (Eckl et al. 1993b, Eckl 1995). These investigations proved not only that primary hepatocytes are also highly sensitive indicators for the genotoxic potential of contaminated surface water samples but additionally indicated that only examining undiluted samples can lead to misinterpretations of the actual situation. Complex environmental mixtures can contain both cytotoxic and genotoxic compounds which as a whole usually do not give linear dose responses. Instead the analysis of dose responses gives either saturation or bell-shaped curves - when different dilutions of the samples are analysed. These investigations further demonstrated that different endpoints of genotoxicity have to be determined, reflecting different modes of action at the DNA level.

The same behaviour was found when applying this test to air and exhaust samples (Eckl et al. 1998, Ares et al. 2000).

\section{Ex vivo/in vitro testing}

The background level of genotoxic effects in primary hepatocytes is higher by a factor of 2 or 3 (depending on the endpoint of genotoxicity) compared to other cellular test systems. Since hepatocytes react very sensitively to mutagens/promutagens it was assumed that dietary intake of mutagens/promutagens could account for this observation. To test this hypothesis rats were fed either a commercial diet or a semi-synthetic diet. After different times of feeding (up to 34 weeks) hepatocytes were isolated, stimulated to proliferate and analysed for genotoxic effects (Eckl et al. 1991). This feeding protocol led to a time-dependent increase of the SCE rate in the animals on a commercial diet while the SCE rate in the animals on the semi-synthetic diet remained constant throughout the feeding period indicating an agedependent accumulation of genetic damages and a contribution to the background SCE rates observed in primary culture. This observation also indicated the suitability of hepatocytes as biological monitors of exposure to mutagens/promutagens via the diet.

This hypothesis was further tested by trapping feral rats - both house rats (Rattus rattus) and Norway rats (Rattus norvegicus) at a waste disposal site. The results of this investigation revealed a weight (age)-dependent increase of chromosomal aberrations which was less pronounced in the Norway rat most probably reflecting the residence time at the waste disposal site. Additionally, this study revealed an age-dependent decrease of chromosomal aberrations in F1 rats housed in the animal facility indicating that the offspring had been exposed transplacentally (Eckl and Riegler 1997).

In summary, primary hepatocytes are suitable and highly sensitive indicators for the cyto- and genotoxic action of xenobiotic substances. The applications range from in vitro testing of potential mutagens/promutagens and complex (environmental) mixtures to ex vivo/in vitro testing of the effects of 
food constituents (Slamenova et al. 2002) and biomonitoring. The range of endpoints that can be analysed is not restricted to those referred to in this paper but includes also endpoints of cytotoxicity such as influences on the proliferation rate and the induction of necrosis and apoptosis.

Received 30 $0^{\text {th }}$ May 2003.

Published online $30^{\text {th }}$ June 2003.

Simultaneously published in J. Berger (ed.): Advances in Cell Biology. Kopp Publ., České Budějovice 2003, p. 157-176.

\section{REFERENCES}

Ares J., P.M. Eckl, I. Raffelsberger: Genotoxicity at low level dose of inspirable urban ambient air particulate in a semiarid regime. Environ. Monit. Assess. 63: 389-408, 2000.

Berry M.N., A.M. Edwards, G.J. Barrit: Isolated hepatocytes. Preparation, properties and applications. Elsevier, Amsterdam-New YorkOxford 1991, pp. 2.

DeFlora S.: Metabolic deactivation of mutagens in the Salmonella-microsome test. Nature 271: 455-456, 1978.

Eckl P.M., W.R. Whitcomb, G. Michalopoulos, R.L. Jirtle: Effects of EGF and calcium on adult parenchymal hepatocyte proliferation. J. Cell. Physiol. 132: 363-366, 1987a.

Eckl P.M., S.C. Strom, G. Michalopoulos, R.L. Jirtle: Induction of sister chromatid exchanges in cultured adult rat hepatocytes by directly and indirectly acting mutagens/carcinogens. Carcinogenesis 8: 10771083, $1987 b$.

Eckl P.M. and H. Esterbauer: Genotoxic effects of 4-hydroxyalkenals. Adv. Biosci 76: 141-157, 1989.

Eckl P.M., T. Alati, R.L. Jirtle: The effects of a purified diet on sister chromatid exchange frequencies and mitotic activity in adult rat hepatocytes. Carcinogenesis 12: 643-646, 1991.

Eckl P.M.: Occurrence and possible consequences of multipolar mitoses in primary cultures of adult rat hepatocytes. J. Cell. Physiol. 154: 601-607, 1993.

Eckl P.M., A. Ortner, H. Esterbauer: Genotoxic properties of 4-hydroxyalkenals and analogous aldehydes. Mutation Res. 290: 183-192, 1993a.

Eckl P.M., L. Anderson-Carnahan, R.L. Jirtle: Aquatic genotoxicity testing with rat hepatocytes in primary culture. I. SCE induction. Sci. total Environ. 136: 111-119, 1993b.

Eckl P.M.: Aquatic genotoxicity testing with rat hepatocytes in primary culture. II. Induction of micronuclei and chromosomal aberrations. Sci. Total Environ. 159: 81-89, 1995.

Eckl P.M. and D. Riegler: Levels of chromosomal damage in hepatocytes of wild rats living within the area of a waste disposal plant. Sci. Total Environ. 196: 141-149, 1997.

Eckl P.M. and I. Raffelsberger: The primary rat hepatocyte micronucleus assay: general features. Mutation Res. 392: 117-124, 1997.

Eckl P.M., P. Leikermoser, M. Wörgetter, H. Prankl, F. Wurst: The mutagenic potential of Diesel and Biodiesel exhausts. In N. Marini and J. Schell (Eds.): Plant Oils as Fuels, Springer Verlag, Berlin-Heidelberg-New York 1998, pp. 123-140.

Fenech M.: The cytokinesis-block micronucleus technique: A detailed description of the method and its application to genotoxicity studies in human populations. Mutation Res. 285: 35-44, 1993.

Guillouzo A.: Use of isolated and cultured hepatocytes for xenobiotic metabolism and cytotoxicity studies. In A. Guillouzo and C. Guguen-Guillouzo (Eds.): Research in Isolated and Cultured Hepatocytes, John Libbey Eurotext Ltd., Montrouge 1986, pp. 313-332.

Hirata K., Y. Yoshida, K. Shiramatsu, A.E. Freeman, H. Hayasaka: Effects of laminin, fibronection and type IV collagen on liver cell cultures. Exp. Cell Biol. 51: 121-129, 1983.

Kremers P.: Drug metabolism in cultured fetal hepatocytes. In A. Guillouzo and C. GuguenGuillouzo (Eds.): Research in Isolated and Cultured Hepatocytes, John Libbey Eurotext Ltd., Montrouge 1986, pp. 285-312.

Maron D.M. and B.N. Ames: Revised methods for the Salmonella mutagenicity test. In B.J. Kilbey, M. Legator, W. Nichols, C. Ramel (Eds.): Handbook of Mutagenicity Test procedures, Elsevier, Amsterdam, pp. 93-141, 1983.

Michalopoulos G., H.D. Cianciulli, A.R. Novotny, A.D. Kligerman, S.C. Strom, R.L Jirtle: Liver regeneration studies with rat hepatocytes in primary culture. Cancer Res. 42: 4673-4682, 1982.

Michalopoulos G: Liver regeneration: molecular mechanisms of growth control. FASEB J. 4: 176-187, 1990.

Natarajan A.T., A.D. Tates, P.P.W. van Buul, M. Meijers, N. de Vogel: Cytogenetic effects of mutagens/carcinogens after activation in a microsomal system in vitro. I. Induction of chromosome aberrations and sister chromatid exchanges by diethylnitrosamine (DEN) and 
dimethylnitrosamine (DMN) in $\mathrm{CHO}$ cells in the presence of rat-liver microsomes. Mutation Res. 37: 83-90, 1976.

Reid L.M., M. Narita, M. Fujita, Z. Murray, C. Liverpool, L. Rosenberg: matrix and hormonal regulation of differentiation in liver cultures. In A. Guillouzo and C. GuguenGuillouzo (Eds.): Research in Isolated and Cultured Hepatocytes, John Libbey Eurotext Ltd., Montrouge 1986, pp. 225-258.

Reisenbichler H. and P.M. Eckl: Genotoxic effects of selected peroxisome proliferators. Mutation Res. 286: 135-144, 1993.

Sattler C.A., N. Sawada, G.L. Sattler, H.C. Pitot: Electron microscopic and time lapse studies of mitosis in cultured rat hepatocytes. Hepatology 8: 1540-1549, 1989.

Slamenova D., I. Chalupa, S. Robichova, A. Gabelova, T. Farkasova, L. Hrusovska,
G. Bacava, L. Sebova, P. Eckl, N. Bresgen, P. Zeitheim, P. Schneider, L. Wsolova, M. Barancokova, A. Kazimirova, J. Navarova, S. Bezek: Effect of dietary intake of vitamin A or E on the level of DNA damage, chromosomal aberrations, and micronuclei induced in freshly isolated rat hepatocytes by different carcinogens. Nutr. Cancer 42: 117-124, 2002.

Strom S.C. and G. Michalopoulos: Mutagenesis and DNA binding of benzo(a)pyrene in co-cultures of rat hepatocytes and human fibroblasts. Cancer Res. 42: 4519-4524, 1982.

Strom S.C., R.L. Jirtle, G. Michalopoulos: Genotoxic effects of 2-acetylaminofluorene on rat and human hepatocytes. Environ. Health Perspect. 49: 165-170, 1983.

\section{* Address:}

Peter M. Eckl, Institute of Genetics and General Biology, University of Salzburg, Hellbrunnerstr. 34, A-5020 Salzburg, Österreich, Peter.Eckl.@sbg.ac.at 